\title{
A cellular spinal cord scaffold seeded with rat adipose-derived stem cells facilitates functional recovery via enhancing axon regeneration in spinal cord injured rats
}

\author{
HONG YIN ${ }^{1}$, TAO JIANG ${ }^{1}$, XI DENG $^{2}$, MIAO YU $^{1}$, HUI XING $^{1}$ and XIANJUN REN ${ }^{1}$ \\ Departments of ${ }^{1}$ Orthopedics and ${ }^{2}$ Ultrasound, Xinqiao Hospital, The Third \\ Military Medical University, Chongqing 400037, P.R. China
}

Received May 21, 2017; Accepted September 7, 2017

DOI: $10.3892 / \mathrm{mmr} .2017 .8238$

\begin{abstract}
Spinal cord injury (SCI), usually resulting in severe sensory and motor deficits, is a major public health concern. Adipose-derived stem cells (ADSCs), one type of adult stem cell, are free from ethical restriction, easily isolated and enriched. Therefore, ADSCs may provide a feasible cell source for cell-based therapies in treatment of SCI. The present study successfully isolated rat ADSCs (rADSCs) from Sprague-Dawley male rats and co-cultured them with acellular spinal cord scaffolds (ASCs). Then, a rat spinal cord hemisection model was built and rats were randomly divided into 3 groups: SCI only, ASC only, and ASC + ADSCs. Furthermore, behavioral tests were conducted to evaluate functional recovery. Hematoxylin \& Eosin staining and immunofluorence were carried out to assess histopathological remodeling. In addition, biotinylated dextran amines anterograde tracing was employed to visualize axon regeneration. The data demonstrated that harvested cells, which were positive for cell surface antigen cluster of differentiation (CD) 29, CD44 and CD90 and negative for CD4, detected by flow cytometry analysis, held the potential to differentiate into osteocytes and adipocytes. Rats that received transplantation of ASCs seeded with rADSCs benefited greatly in functional recovery through facilitation of histopathological rehabilitation, axon regeneration and reduction of reactive gliosis. rADSCs co-cultured with ASCs may survive and integrate into the host spinal cord on day 14 post-SCI.
\end{abstract}

\footnotetext{
Correspondence to: Dr Xianjun Ren, Department of Orthopedics, Xinqiao Hospital, The Third Military Medical University, 183 Xinqiao Street, Chongqing 400037, P.R. China

E-mail: ren_xianjun@sina.com
}

Key words: acellular spinal cord scaffold, adipose-derived stem cells, spinal cord injured, axon regeneration, reactive gliosis

\section{Introduction}

Spinal cord injury (SCI), usually resulting in severe sensory and motor deficits, is a major public health concern due to high disability rate and low curable rate (1). In the past decades, though a number of therapeutic strategies have been developed, such as medication, surgical intervention and even gene therapy, they provide little benefits for patients with SCI (2). To date, tissue engineering sheds light on a promising therapeutic approach for treating SCI resulting from priority to provide a suitable niche for survival and functional exertion of local residued neural cell types in injuried spinal cord.

Especially, transplantation of tissue engineering materials seeded with stem cells has proven to benefit functional recovery through promoting axonal extension, immunosuppression and/or neurotrophic support (3-5). Our colleagues have developed a method to obtain modified acellular spinal cord scaffolds (ASCs), which is natural, soft, flexible and stable under physiological conditions. Meanwhile, it contains linear guidance pores extending through their full Iength, which could serve as a support for axon regeneration. In addition, we have seeded bone marrow mesenchymal stem cells (BMSCs) in ASCs to certify their biocompatibility in vitro $(6,7)$. Furthermore, studies have indicated that engraftment of ASCs seeded with umbilical cord blood-derived mesenchymal stem cells (UCB-MSCs) help enhance functional recovery via bridging spinal cord cavity and promoting long-distance axon regeneration in SCI rats (8). In addition, the lack of integrality of umbilical cord database and long-time storage under frozen condition might be limitations to restrict the autologous transplantation of UCB-MSCs in ready-to-use clinical application. Hence, it is significant to find out a ready-to-use autologous stem cell resource for engraftment.

Adipose-derived stem cells (ADSCs), identified as a source of multipotent cells (9), possess the potential to protect neural cells from glutamate excitotoxicity-induced apoptosis (10), differentiate into glial cells, secrete neurotrophic factors $(11,12)$ and suppress local inflammation after central nervous system (CNS) injury (13). Meanwhile, transplantation of ADSCs, which could be easily isolated from abundant adipose tissue in the same subject with less damage, helps improve mechanical allodynia and functional recovery in 
rats with injured sciatic nerve (14). In addition, recent studies have focused on transplantation of ADSCs with other effective therapies to ensure its application in therapeutic strategy for SCI $(15,16)$. ASC, a candidate for treatment of SCI, is worth illustrating its application combined with ASDCs and possible underlying mechnism.

In the present study, we hypothesized that transplantation of ASCs seeded with rat ADSCs (rADSCs) would facilitate functional recovery via harnessing axon regeneration and reducing reactive gliosis in injuried rat spinal cord. To test our hypothesis, we cultured rADSCs and assessed their characteristics. Meanwhile, the biocompatibility between rADSCs and ASCs was evaluated using co-culture pattern and Brdu assays in vitro and in vivo. In addition, the beneficial ability to restore injuried spinal cord of ASCs seeded with rADSCs was examined through histopathological assessment using $\mathrm{HE}$, immunofluorescence staining, and behavioral test. The aim of this study is to provide suitable strategy for SCI treatment based on tissue engineering, at the same time, to probably enlarge the application scope of ADSCs from bench to bed in the future research.

\section{Materials and methods}

Cell culture. rADSCs were obtained according to the previous protocols $(11,17,18)$. Briefly, the adipose tissues were dissected from subcutaneous fat in the inguinal region of Sprague-Dawley male rats. The tissues were washed three times with phosphate-buffered saline (PBS) and minced with scissors. Then, tissues were digested in PBS containing $2 \mathrm{mg} / \mathrm{ml}$ collagenase type II (Sigma-Aldrich, St. Louis, MO) for $40 \mathrm{~min}$ at $37^{\circ} \mathrm{C}$ with constant agitation. Then, tissues was mixed with an equal volume of Dulbecco's Modified Eagle's Medium (DMEM, Gibco, Grand Island, NY) supplemented with $10 \%$ fetal bovine serum (FBS, Gibco, Grand Island, NY), passed through a $40-\mu \mathrm{m}$ Nylon cell strainer (BD Falcon, San Jose, CA) and centrifuged for $10 \mathrm{~min}$ at 1,500 g. Afterward, floating adipocytes were removed from the stromal vascular fraction. Finally, the obtained cells were seeded on cell culture flasks (Thermo Fisher Scientific, Waltham, MA) supplemented with $\alpha$-modified Eagle's medium (Gibco, Grand Island, NY), $10 \%$ FBS, and 1\% penicillin-streptomycin (Gibco, Grand Island, NY). They were incubated at $37^{\circ} \mathrm{C}$ in a humidified atmosphere under $5 \% \mathrm{CO}_{2}$. The culture medium was replaced every 2-3 days. Cells were trypsinized with $0.25 \%$ trypsin-EDTA (Gibco, Grand Island, NY) and expanded in new flasks when they reached $70-80 \%$ confluence. Cells used for transplantation in ASCs were from passages 3 to 6 .

Differentiation assay. For osteogenic differentiation, rADSCs at passage 3 were inoculated at $4 \times 10^{3}$ cells $/ \mathrm{cm}^{2}$ and cultured for 2 weeks in osteogenic induction medium [MEM containing $10 \% \mathrm{FBS}, 100 \mu \mathrm{g} / \mathrm{ml}$ ascorbate, $0.1 \mu \mathrm{M}$ dexamethasone and $10 \mathrm{mM} \beta$-glycerophosphate (All from Cyagen Biosciences, Suzhou, Jiangsu, China)]. rADSCs were then fixed with $4 \%$ paraformaldehyde (PFA, Boster Biosciences, Beijing, China) for $20 \mathrm{~min}$, washed with PBS for 3 times, afterward, incubated with $1 \mathrm{mg} / \mathrm{ml}$ Alizarin Red (Sigma-Aldrich, St. Louis, MO) solution for $30 \mathrm{~min}$ to stain for calcium deposition. For adipogenic differentiation, rADSCs at passage 3 were cultured for 2 weeks in adipogenic induction medium [high glucose-DMEM containing 10\% FBS, $1 \mu \mathrm{M}$ dexamethasone, $0.5 \mathrm{mM}$ methyl-isobutylxanthine, $10 \mu \mathrm{g} / \mathrm{ml}$ insulin, and $100 \mu \mathrm{M}$ indomethacin (All from Cyagen Biosciences, Suzhou, Jiangsu, China)]. Then, cells were fixed with $4 \%$ PFA for 20 min, washed with PBS for 3 times, afterward, immersed in $0.3 \%$ Oil Red O solution (Sigma-Aldrich, St. Louis, MO) in $60 \%$ isopropanol for $30 \mathrm{~min}$ to assess lipid droplet formation.

Flow cytometry analysis. Surface antigens of the undifferentiated rADSCs were identified by flow cytometry using anti-CD29 (1:200, Biolegend, San Diego, CA), CD90 (1:100, Biolegend, San Diego, CA), CD44 (1:400, Abcam, Cambridge, UK) and CD45 (1:200, Biolegend, San Diego, CA) antibodies. rADSCs were resuspended in buffer containing PBS supplemented with $1 \%$ bovine serum albumin (BSA, Gibco, Grand Island, NY) to reach a concentration of $1 \times 10^{7}$ cells $/ \mathrm{ml}$ after trypsinizing with $0.25 \%$ trypsin-EDTA, and incubated with fluorescence-conjugated primary antibodies for $30 \mathrm{~min}$ on ice. Flow cytometry analysis was performed on the BD Accuri $^{\mathrm{TM}}$ C6 Flow Cytometer System (BD Biosciences, NJ, USA) and data were analyzed using Cflow Plus software (Becton-Dickinson, NJ, USA).

Preparation of rat acellular spinal cord scaffolds. The rat ASCs were harvested as previously described according to standard procedures developed by our colleagues $(6,7)$. In short, SD rats were anesthetized with intraperitoneal injection of sodium pentobarbital ( $45 \mathrm{mg} / \mathrm{kg}$ body weight). Thoracic spinal cord samples $(\sim 20 \mathrm{~mm})$ were obtained and then rinsed in PBS to remove blood. Obtained tissues were incubated in distilled water (changed per $2 \mathrm{~h}$ ) at room temperature for $6 \mathrm{~h}$. Then, samples were serially immersed in $1 \%$ Triton X-100 solution ( $3 \mathrm{~h})$, PBS ( $3 \mathrm{~h}), 1 \%$ sodium deoxycholate solution $(3 \mathrm{~h})$, and PBS ( $3 \mathrm{~h})$, respectively. Meanwhile, specimens were oscillated at $100 \mathrm{rpm}$ throughout the immersion process. Afterward, samples were lyophilized to obtain the acellular spinal cord scaffolds after serial immersions were repeated once. The lyophilized scaffolds were incubated in genipin and glutaraldehyde solutions (both $5 \mathrm{mg} / \mathrm{ml}$ in $0.01 \mathrm{M} \mathrm{PBS}$, $\mathrm{pH}$ 7.4), respectively, for chemical crosslinking for $24 \mathrm{~h}$ at $37^{\circ} \mathrm{C}$. All specimens were immediately freeze-dried for $24 \mathrm{~h}$ in freeze drier and sterilized by irradiation (16 kGy) with Cobalt-60 gamma rays before engraftment. Before transplantation, rADSCs were cultured in $10 \mu \mathrm{M}$ 5-bromodeoxyuridine (BrdU, Sigma-Aldrich, St. Louis, MO) for 48 h. Subsequently, they were injected into ASCs $(0.2 \times 0.1 \mathrm{~cm})$ at a concentration of $5 \times 10^{6}$ cells $/ \mathrm{ml}$. The engraftments were then incubated at $37^{\circ} \mathrm{C}$ in a humidified atmosphere under $5 \% \mathrm{CO}_{2}$ in rADSCs culture medium for 2-3 days, stained with hematoxylin and eosin (HE), and examined by light microscopy. The ultrastructure of the engraftment were visualized by scanning electron microscopy (SEM, S-3400 N, Hitachi, Japan) (7).

Rat spinal cord hemisection and transplantation. All animal experiments were approved and supervised by the ethics committee of The Third Military Medical University according to international standards for animal welfare. Adult male SD rats (body weight, 200-250 g) were purchased from animal center of The Third Military Medical University 
and anesthetized with intraperitoneal injection of sodium pentobarbital ( $45 \mathrm{mg} / \mathrm{kg}$ body weight). Thoracic spinal cord segments were exposed after laminectomy at the T9-10 level under aseptic condition. Two right-sided hemisections of spinal cord were performed by microdissection scissor between T9 and T10 level under surgical microscope. A 2 mm-width gap was created with a 22 -gauge syringe needle (8). Then, muscle layers and skin were sutured separately. To prevent infection, rats were subcutaneously injected with ampicillin $(100 \mathrm{mg} / \mathrm{kg})$ and gentamicin $(12 \mathrm{mg} / \mathrm{kg})$ following 3 days after surgery (once a day). Their bladders were emptied twice daily until they regained bladder control. Rats were randomly divided into 3 groups after hemisected SCI: (1) without any intervention (SCI only, $\mathrm{n}=15$ ), (2) with ASC scaffolds implantation (ASC only, $\mathrm{n}=15$ ), (3) with ASC scaffolds seeded with rADSCs transplantation (ASC + ADSCs, n=15). Behavioral tests (including BBB locomotor rating scale, hypersensitivity to mechanical and thermal stimulation) were assessed weekly before and after surgery. Histological examinations and immunohistochemistry were performed on all animals. BDA anterograde tracing were used to detect nerve fibers in injuried rat spinal cord on day 14 post-SCI.

Behavioral tests after SCI. For bsehavioral tests, two independent investigators blinded to the experimental design separately assessed and scored behavioral recovery. The locomotor recovery after SCI was evaluated in view of the BBB score as previously described (19) on day 1, 7, 14, 21 and 28 post-SCI.

$B D A$ anterograde tracing. Rats were anesthetized and placed on a stereotaxic frame and biotinylated dextran amines (BDA, Invitrogen, Breda, Netherlands) was injected in view of previous study before conducting surgery at spinal cord (20).

Hematoxylin and eosin $(H \& E)$ staining. Scaffolds and tissue sections were fixed in $4 \%$ paraformaldehyde, paraffin-embedded, sliced, and stained with hematoxylin and eosin (H\&E, Beyotime C0105, Beijing, China) according to the manufacture's instructions (21). The stained samples were visualized using a light microscopy (Leica, Wetzlar, Germany).

Histological analysis. The histological analysis was employed according to standard procedures (8) and the antibodies were as follows: Mouse anti-Brdu (1:200, Abcam, Cambridge, UK), rabbit anti-glial fibrillary acidic protein (GFAP, 1:300, Abcam, Cambridge, UK).

For Brdu staining, sections were firstly incubated in $2 \mathrm{M}$ $\mathrm{HCL}$ for $30 \mathrm{~min}$, then neutralized with $0.1 \mathrm{M}$ sodium borate buffer $(\mathrm{pH} 8.5)$ for $30 \mathrm{~min}$ at room temperature for DNA hydrolysis (22). Slices were visualized by a light microscopy (Leica, Wetzlar, Germany).

For BDA tracing, three sagittal slices were selected from each group and rinsed with PBS , then treated with avidin-Cy3 containing $0.25 \%$ TritonX-100 in PBS at room temperature for $2 \mathrm{~h}$ and rinsed in PBS for three times for analysis.

All sections with immunofluorescence were examined by a confocal microscope (Carl Zeiss, LSM780, Weimar, Germany) and analyzed using Zen 2011 software (Carl Zeiss, Weimar, Germany).
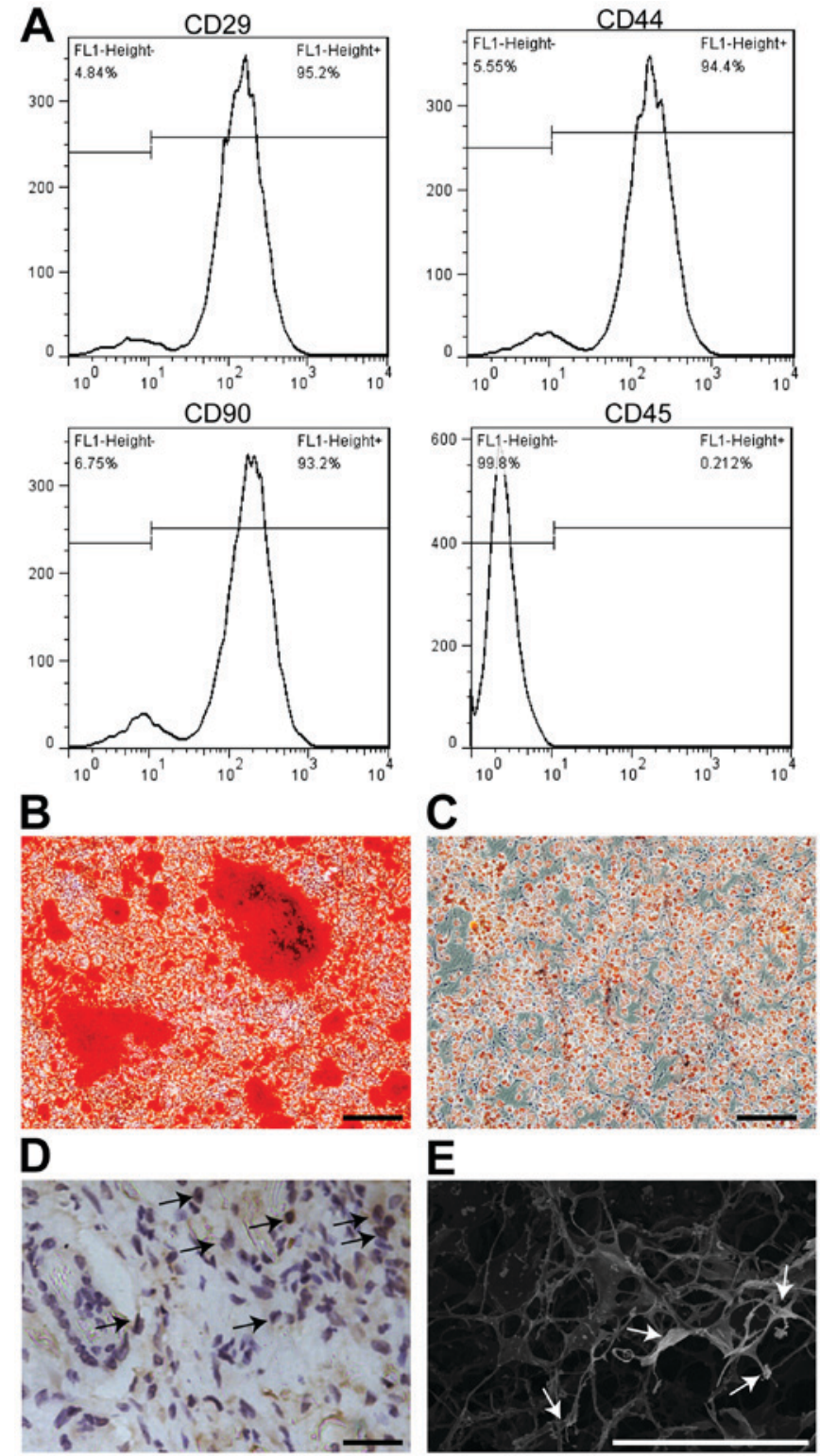

Figure 1. Characterization of rADSCs and biocompatibility of ASCs. (A) Cell surface antigen analysis of rADSCs at passage 3 identified cultured cells were positive for CD29, CD44 and CD90, but negative for CD45. (B) Osteogenesis potential of rADSCs was shown by calcification deposition (Alizarin Red stain). (C) Adipogenesis feature of rADSCs was represented by lipid droplet formation (Oil Red O staining). (D) Immunostaining illustrated Brdu-labeled rADSCs (arrows) interspersed in ASCs before transplantation. (E) SEM of ASCs seeded with rADSCs before engraftment. Scale bars, $100 \mu \mathrm{m}$.

Statistical analysis. Data were represented as the mean \pm SEM and data analysis were conducted using SPSS V18.0 (SPSS Inc, Chicago, IL). Comparisons among multiple sets were performed using One-way ANOVA followed by Tukey's post hoc test and a $\mathrm{P}<0.05$ was considered as significance.

\section{Results}

Characterization of rADSCs and biocompatibility of ASCs. rADSCs expressed cell surface antigen CD29, CD44 and CD90 after 3 passages, but negative for CD 45 by flow cytometry analysis (Fig. 1A). To assess the differentiation potential of cultured cells, they were incubated in induction media for 14 day 
to evaluate their differentition into osteocytes and adipocytes, respectively. The results indicated that cultured cells held the capability of osteogenesis (Fig. 1B) and adipogenesis (Fig. 1C). To test the biocompatibility of ASCs, rADSCs pre-cultured in $10 \mu \mathrm{M}$ BrdU were seeded into ASCs, which appeared to be white color and became soft and flexible two days latter. Brdu ${ }^{+}$ cells were interspersed in the network of ASCs before transplantation (Fig. 1D). Meanwhile, rADSCs with thin fibers forming uniform mesh pores were observed using SEM (Fig. 1E). In short, these results indicated rADSCs were able to survive and exert biological function in ASCs, which were safe and biocompatible, according to our established procedures.

Transplantation of ASCs seeded with rADSCs promoted functional recovery after SCI in rats. To explore the functional benefits with ASCs seeded with rADSCs, BBB scores, hypersensitivity to mechanical and thermal stimulation assays were performed on day 1, 7, 14, 21 and 28 post-SCI in three groups: SCI only, ASC only and ASC + ADSCs. The data represented that rats in ASC + ADSCs group showed the best improvement in locomotor recovery. Meanwhile, rats in ASC only group represented better than those in SCI only group from day 14 after SCI (Fig. 2A). Moreover, rats in ASC + ADSCs group depicted the same trend as locomotor renovation in the other two functional tests of hypersensitivity to mechanical and thermal stimulation assays (Fig. 2B and C). Together, the functional tests data demonstrated that implantation of ASCs seeded with rADSCs was able to enhance functional recovery after SCI in rats.

Transplantation of ASCs seeded with rADSCs facilitated histopathological rehabilitation. To illuminate the reason why functional recovery improved greatly in rats receiving engraftment of ASCs seeded with rADSCs, H\&E staining was used to observe histopathological changes on day 14 post-SCI. The images represented that rats in ASC + ADSCs group showed significant diminution and less atrophy of the injured site than that in the ASConly and SCI only groups (Fig. 3B). Meanwhile, the semi-quantitative data revealed the lesion area in ASC + ADSCs group decreased most than that in the ASC only and SCI only groups. Furthermore, ASC only group indicated better improvement than that in the SCI only group (Fig. 3C). In addition, a certain number of $\mathrm{Brdu}^{+} \mathrm{rADSCs}$ survived in the grafted ASCs and distributed over the host spinal cord in ASC + ADSCs group on day 14 after SCI (Fig. 3D), which was in line with previous study in hUCB-MSCs transplantation (8).

Transplantation of ASCs seeded with rADSCs promoted axon regeneration and reduced reactive gliosis. Our previous research implied that ASCs manufactured by our colleagues might potentially support axon regeneration after nervous system injury (6). Here, we conducted BDA anterograde tracing with immunofluorescence staining showing that transplantation of ASCs seeded with rADSCs held the best improvement potential in harnessing axon regeneration to bridge lesions in ASC + ADSCs group than that in the ASC only and SCI only groups. BDA positive area in ASC only group was larger than that in the SCI only group (Fig. 4A). Meanwhile, the semi-quantitative data indicated similar tendency observed from Fig. 4A (Fig. 4B). Moreover, we performed GFAP staining to assess
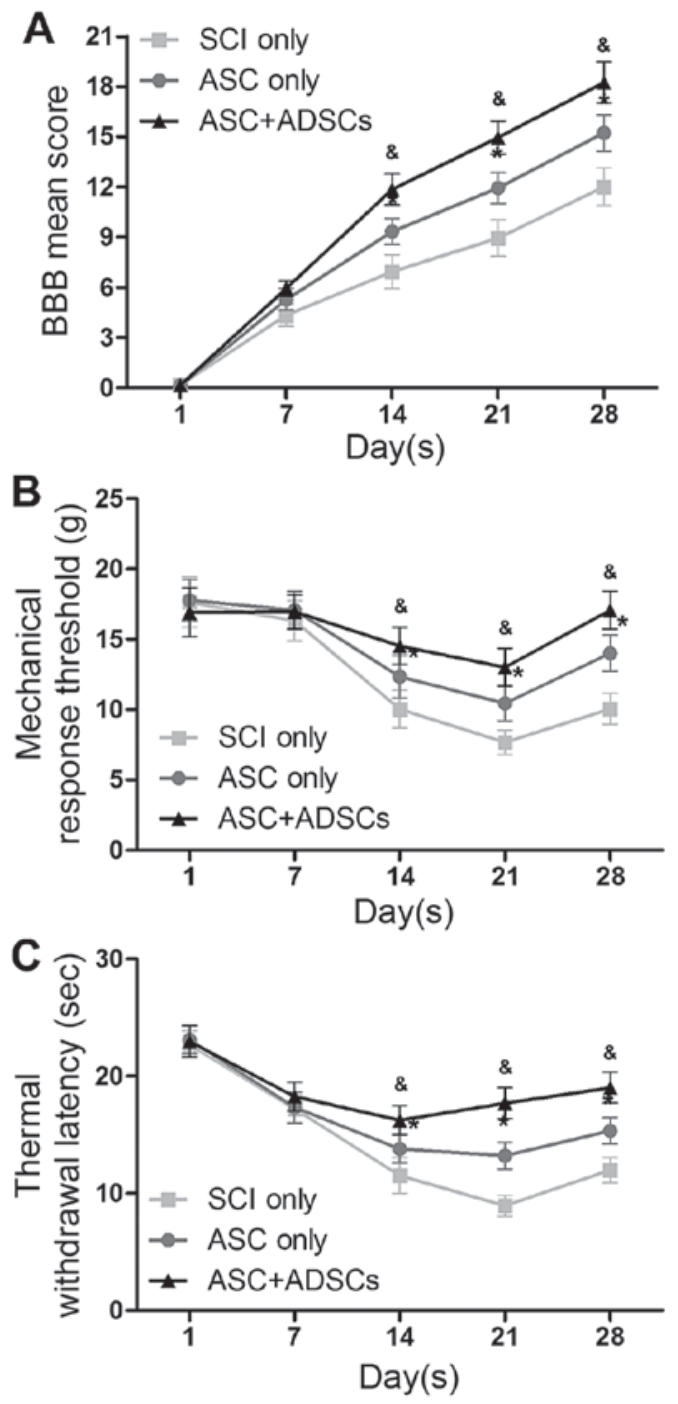

Figure 2. Transplantation of ASCs seeded with rADSCs promotes functional recovery. (A) Transplantation of ASCs seeded with rADSCs promoted hindlimb motor functional rehabilitation in rats in ASC + ADSCs group, compared with SCI and ASC only groups from day 14 post-SCI (SCI only, $\mathrm{n}=15$; ASC only, $\mathrm{n}=15$; ASC + ADSCs, $\mathrm{n}=15$ ). (B and C) Hypersensitivity to mechanical and thermal stimulation induced by SCI was greatly alleviated in ASC + ADSCs group, compared with SCI and ASC only groups from day 14 post-SCI. ${ }^{*} \mathrm{P}<0.05$ compared with $\mathrm{SCI}$ only group, ${ }^{\&} \mathrm{P}<0.05$ compared with ASC + ADSCs group. One-way ANOVA followed by Tukey's post hoc test. g, gramma; sec, second.

reactive gliosis in lesions. The results indicated that $\mathrm{GFAP}^{+}$area was much smaller in ASC + ADSCs group than that in the ASC only and SCI only groups (Fig. 4C and D). Furthmore, there was no obvious difference between ASC only group and SCI only group (Fig. 4C and D). Taken together, our data certified that ASCs manufactured by our colleagues could support axon regeneration and implantation with ADSCs enhanced this effectiveness after SCI. In addtion, it reduced reactive gliosis, and might finally decreased glial scar formation when transplantation ASCs seeded with ADSCs.

\section{Discussion}

In the present study, data indicated that ASCs manufactured according to our procedures held the ability to promote axon 


\section{A}
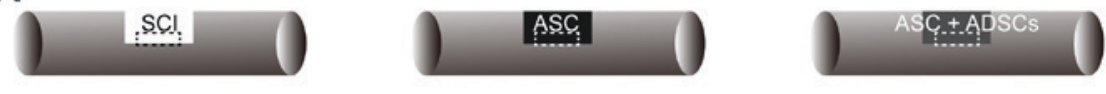

B

14 days post-SCl

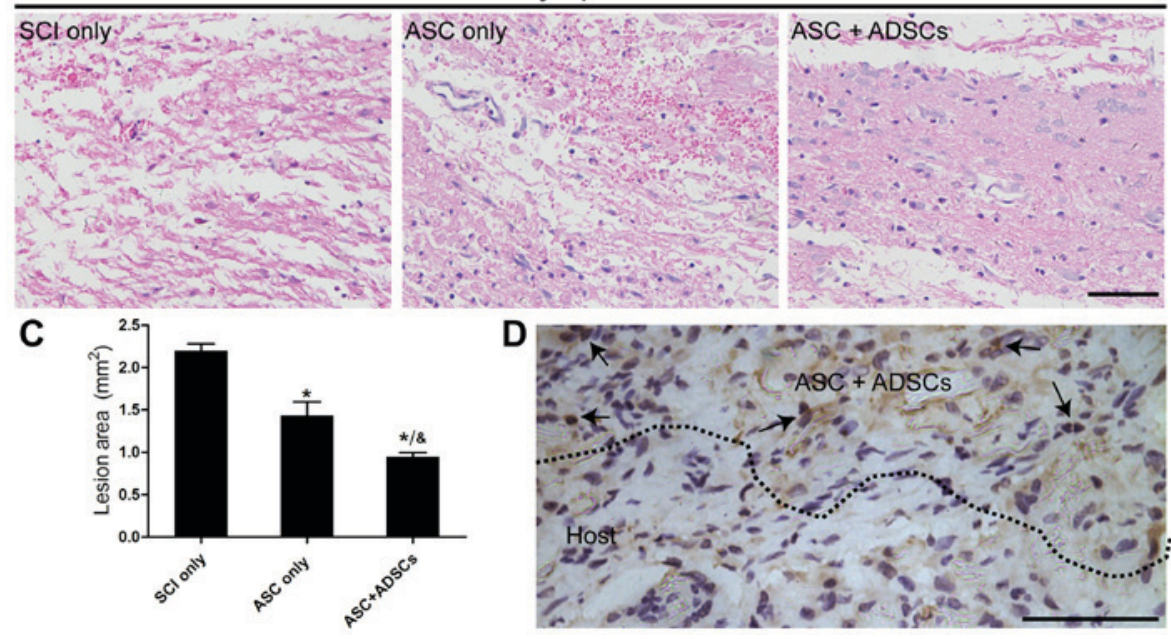

Figure 3. Transplantation of ASCs seeded with rADSCs facilitates injuried spinal cord renovation. (A) Schematic drawings of spinal cord and regions for transplantation of ASCs to bridge hemisected lesion gap. Dotted rectangle indicated the tissue region examined at histopathological level. (B) Sagittal section of HE staining from SCI only, ASC only and ASC + ADSCs groups on day 14 after SCI, respectively. (C) Semi-quantification of hemisected spinal cord lesions in view of (B). (D) A number of Brdu-labeled cells (arrows) were shown in the host spinal cord and ASCs on day 14 post-SCI. The dotted lines identified the injuried spinal cord from engrafted ASCs. "P $<0.05$ compared with $\mathrm{SCI}$ only group, ${ }^{\circledR} \mathrm{P}<0.05$ compared with ASC + ADSCs group. One-way ANOVA followed by Tukey's post hoc test. Scale bars, $100 \mu \mathrm{m}$.
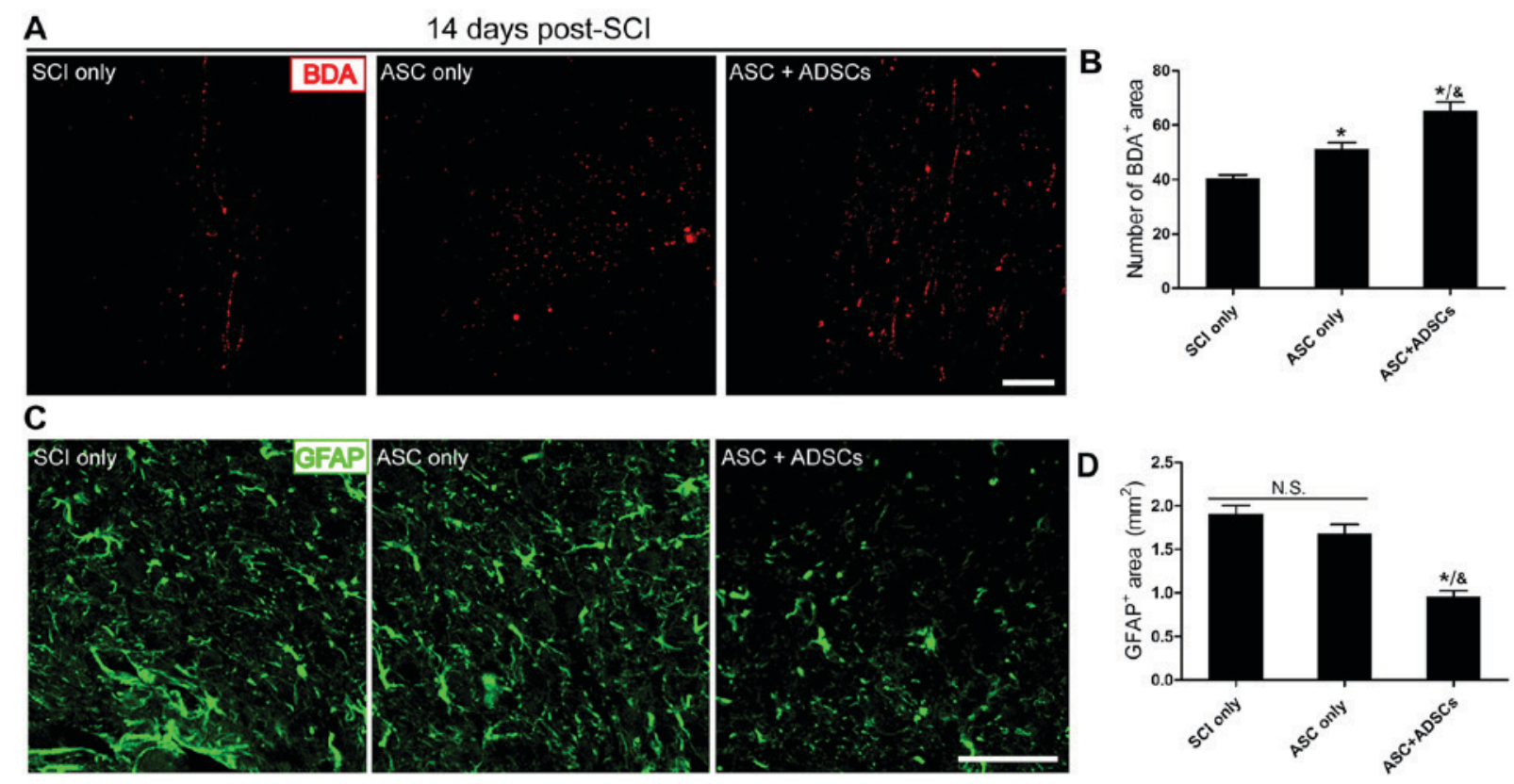

Figure 4. Transplantation of ASCs seeded with rADSCs harnesses axon regeneration and reduces reactive gliosis. (A) Immunofluorescence represented BDA staining in red channel from SCI only, ASC only and ASC + ADSCs groups on day 14 after SCI, respectively. (B) Semi-quantification of BDA expression according to immunostaining (A). (C) Immunofluorescence demonstrated GFAP staining in green channel from SCI only, ASC only and ASC + ADSCs groups on day 14 after SCI, respectively. (D) Semi-quantification of GFAP expression according to immunostaining (B). "P<0.05 compared with SCI only group, ${ }^{\&} \mathrm{P}<0.05$ compared with ASC + ADSCs group. One-way ANOVA followed by Tukey's post hoc test. N.S. indicates no significant difference.

regeneratin in vivo, which enlarged the application of ASCs based on our previous studies (6,7). Here, we also introduced a new stem cell type, named ADSCs, for transplantation in treatment of SCI, especially engraftment with ASCs. Meanwhile, we preliminarily explored the underlying mechanism, and results revealed that ASCs seeded with rADSCs facilitated histopathological rehabilitation and axon regeneration, while reduced reactive gliosis. Furthermore, our data indicated that rADSCs were biocompatible with host spinal cord tissue, which might be served as a cause resulting in functional improvement, and survived rADSCs might guide axons sprouting into the distal lesions in injuried spinal cord, 
therefore ultimately reinforced effective reinnervation of target neurons.

ADSCs, one type of adult stem cells, are free from ethical restriction associated with embryonic stem cells (ES) or neural stem cells (NSCs) $(23,24)$. Furthermore, ADSCs are easily isolated and enriched from abundant adipose tissue in the same subject with less damage, therefore, ADSCs are more suitable for autologous transplantation (25). In addition, research has proved that ADSCs easily escape from immune system surveillance like $\mathrm{T}$ cells because of their surface antigens (14). As a result, ADSCs could serve as an appropriate candidate for autografts, allografts, and even xenografts $(14,26)$. The beneficial effect resulting from implantation of ASCs seeded with rADSCs needs to be explained.

Reasons why implantation of ASCs seeded with rADSCs promote axon regeneration and reduce reactive gliosis can be ascribed as follows: (1) rebalance of local disturbed niche, (2) physical support of allograft, (3) neurotrophic factors secretion and (4) inflammation suppression. Excitotoxic damage, high levels of the gelatinase and matrix metalloproteinase- 9 (MMP-9) often damages local microenvironment in acute phase after SCI to elicit neuronal loss and severe locomotor impairment $(27,28)$. While, inflammation in subacute phase usually increases vascular permeability and myeloid cell infiltration to deteriorate locomotor networks (29-32). Besides, glial scar formation is likely to jeopardize axon outgrowth in chronic phase $(33,34)$. To conquer these factors above and look for a suitable candidate to deal with them once for all, previous studies have revealed that implantation of ADSCs rebuilds the local microenvironment by secreting neurotrophins like brain derived neurotrophic factor (BDNF), nerve growth factor (NGF), basic fibroblasts growth factor (bFGF) and neurogenin $2(35,36)$ after CNS injury. Meanwhile, study also represents ADSCs facilitates laminin secretion, which help restore local blood vessels network (37). Moreover, researchers have also discovered engraftment of ADSCs could suppress local inflammation after stroke $(38,39)$.

There must be some significant issues need to be fully addressed in our future work. First, the mechanism of how transplantation of ASCs seeded with ADSCs to sprout axon outgrowth and the extent of axon outgrowth. Next, the function of ADSCs implantation must be multifaceted, it is better to elucidate which one is dominated in the recovery process. Third, to pursuit excellent effectiveness, therapeutic window for engraftment needs to be optimized as well. These are all vital questions need to be issued in our future work.

In this study, we have demonstrated that transplantation of ASCs seeded with ADSCs is a safe and feasible strategy for cell replacement therapy in the treatment of SCI in rats. The underlying mechanisms must be multifaceted, but at least promoting axon outgrowth and reducing reactive gliosis are two main factors to benefit functional renovation. Given that ADSCs are easily harvested from abundant adipose tissue in the same subject with less damage, so they hold a significant priority of ethical restriction. Furthermore, our results indicate the use of ADSCs is safe and effective, especially transplantation with ASCs, which implies that the engraftment of ASCs seeded with ADSCs could be a feasible therapeutic strategy for SCI.

\section{Acknowledgements}

This study was financed by grants from the National Natural Science Foundation of China $(81271362,81471262)$.

\section{References}

1. Selvarajah S, Hammond ER and Schneider EB: Trends in traumatic spinal cord injury. Jama 314: 1643, 2015.

2. Rubiano AM, Carney N, Chesnut R and Puyana JC: Global neurotrauma research challenges and opportunities. Nature 527 (Suppl): S193-S197, 2015.

3. Requejo-Aguilar R, Alastrue-Agudo A, Cases-Villar M, Lopez-MocholiE, England R, Vicent MJ and Moreno-Manzano V: Combined polymer-curcumin conjugate and ependymal progenitor/stem cell treatment enhances spinal cord injury functional recovery. Biomaterials 113: 18-30, 2017.

4. Ban DX, Liu Y, Cao TW, Gao SJ and Feng SQ: The preparation of rat's acellular spinal cord scaffold and co-culture with rat's spinal cord neuron in vitro. Spinal cord 55: 411-418, 2017.

5. Uchida S, Hayakawa K, Ogata T, Tanaka S, Kataoka K and Itaka K: Treatment of spinal cord injury by an advanced cell transplantation technology using brain-derived neurotrophic factor-transfected mesenchymal stem cell spheroids. Biomaterials 109: 1-11, 2016.

6. Guo SZ, Ren XJ, Wu B and Jiang T: Preparation of the acellular scaffold of the spinal cord and the study of biocompatibility. Spinal Cord 48: 576-581, 2010.

7. Jiang T, Ren XJ, Tang JL, Yin H, Wang KJ and Zhou CL: Preparation and characterization of genipin-crosslinked rat acellular spinal cord scaffolds. Mater Sci Eng C Mater Biol Appl 33: 3514-3521, 2013.

8. Liu J, Chen J, Liu B, Yang C, Xie D, Zheng X, Xu S, Chen T, Wang L, Zhang Z, et al: Acellular spinal cord scaffold seeded with mesenchymal stem cells promotes long-distance axon regeneration and functional recovery in spinal cord injured rats. J Neurol Sci 325: 127-136, 2013.

9. Wu SH, Huang SH, Lo YC, Chai CY, Lee SS, Chang KP, Lin SD, Lai CS, Yeh JL and Kwan AL: Autologous adipose-derived stem cells attenuate muscular atrophy and protect spinal cord ventral horn motor neurons in an animal model of burn injury. Cytotherapy 17: 1066-1075, 2015.

10. Lu S, Lu C, Han Q, Li J, Du Z, Liao L and Zhao RC: Adipose-derived mesenchymal stem cells protect $\mathrm{PC} 12$ cells from glutamate excitotoxicity-induced apoptosis by upregulation of XIAP through PI3-K/Akt activation. Toxicology 279: 189-195, 2011.

11. Tomita K, Madura T, Sakai Y, Yano K, Terenghi G and Hosokawa K: Glial differentiation of human adipose-derived stem cells: Implications for cell-based transplantation therapy. Neuroscience 236: 55-65, 2013.

12. Lukovic D, Stojkovic M, Moreno-Manzano V, Jendelova P, Sykova E, Bhattacharya SS and Erceg S: Concise review: Reactive astrocytes and stem cells in spinal cord injury: Good guys or bad guys? Stem Cells 33: 1036-1041, 2015.

13. Kim Y, Jo SH, Kim WH and Kweon OK: Antioxidant and anti-inflammatory effects of intravenously injected adipose derived mesenchymal stem cells in dogs with acute spinal cord injury. Stem Cell Res Ther 6: 229, 2015.

14. Lee HY, Lee HL, Yun Y, Kim JS, Ha Y, Yoon DH, Lee SH and Shin DA: Human adipose stem cells improve mechanical allodynia and enhance functional recovery in a rat model of neuropathic pain. Tissue Eng Part A 21: 2044-2052, 2015.

15. Sarveazad A, Babahajian A, Bakhtiari M, Soleimani M, Behnam B, Yari A, Akbari A, Yousefifard M, Janzadeh A, Amini $\mathrm{N}$, et al: The combined application of human adipose derived stem cells and chondroitinase $\mathrm{ABC}$ in treatment of a spinal cord injury model. Neuropeptides 61: 39-47, 2017.

16. Ji WC, Zhang XW and Qiu YS: Selected suitable seed cell, scaffold and growth factor could maximize the repair effect using tissue engineering method in spinal cord injury. World J Exp Med 6: 58-62, 2016.

17. Komiyama S, Sakakura C, Murayama Y, Komatsu S, Shiozaki A, Kuriu Y, Ikoma H, Nakanishi M, Ichikawa D, Hujiwara H, et al: Adipose-derived stem cells enhance tissue regeneration of gastrotomy closure. J Surg Res 185: 945-952, 2013. 
18. Choron RL, Chang S, Khan S, Villalobos MA, Zhang P, Carpenter JP, Tulenko TN and Liu Y: Paclitaxel impairs adipose stem cell proliferation and differentiation. J Surg Res 196: 404-415, 2015

19. Basso DM, Beattie MS and Bresnahan JC: Graded histological and locomotor outcomes after spinal cord contusion using the NYU weight-drop device versus transection. Exp Neurol 139: 244-256, 1996

20. Yuan J, Zou M, Xiang X, Zhu H, Chu W, Liu W, Chen F and Lin J: Curcumin improves neural function after spinal cord injury by the joint inhibition of the intracellular and extracellular components of glial scar. J Surg Res 195: 235-245, 2015.

21. Hu R, Zhou J, Luo C, Lin J, Wang X, Li X, Bian X, Li Y, Wan Q, $\mathrm{Yu}$ Y and Feng H: Glial scar and neuroregeneration: Histological, functional and magnetic resonance imaging analysis in chronic spinal cord injury. J Neurosurg Spine 13: 169-180, 2010.

22. Prodromidou K, Papastefanaki F, Sklaviadis T and Matsas R: Functional cross-talk between the cellular prion protein and the neural cell adhesion molecule is critical for neuronal differentiation of neural stem/precursor cells. Stem Cells 32: 1674-1687, 2014.

23. Ide $\mathrm{C}$, Nakano $\mathrm{N}$ and Kanekiyo K: Cell transplantation for the treatment of spinal cord injury-bone marrow stromal cells and choroid plexus epithelial cells. Neural Regen Res 11: 1385-1388, 2016.

24. Nandoe Tewarie RD, Hurtado A, Levi AD, Grotenhuis JA and Oudega M: Bone marrow stromal cells for repair of the spinal cord: Towards clinical application. Cell Transplantat 15: 563-577, 2006.

25. Frese L, Dijkman PE and Hoerstrup SP: Adipose tissue-derived stem cells in regenerative medicine. Transfus Med Hemother 43: 268-274, 2016.

26. Bai L, Lennon DP, Eaton V, Maier K, Caplan AI, Miller SD and Miller RH: Human bone marrow-derived mesenchymal stem cells induce Th2-polarized immune response and promote endogenous repair in animal models of multiple sclerosis. Glia 57: 1192-1203, 2009.

27. Mazzone GL, Veeraraghavan P, Gonzalez-Inchauspe C, Nistri A and Uchitel OD: ASIC channel inhibition enhances excitotoxic neuronal death in an in vitro model of spinal cord injury. Neuroscience 343: 398-410, 2017.

28. Hansen CN, Norden DM, Faw TD, Deibert R, Wohleb ES Sheridan JF, Godbout JP and Basso DM: Lumbar myeloid cell trafficking into locomotor networks after thoracic spinal cord injury. Exp Neurol 282: 86-98, 2016.

29. Anwar MA and Eid AH: Determination of vascular reactivity of middle cerebral arteries from stroke and spinal cord injury animal models using pressure myography. Methods Mol Biol 1462: 611-624, 2016.
30. Lee JY, Na WH, Choi HY, Lee KH, Ju BG and Yune TY: Jmjd3 mediates blood-spinal cord barrier disruption after spinal cord injury by regulating MMP-3 and MMP-9 expressions. Neurobiol Dis 95: 66-81, 2016

31. Haan N, Zhu B, Wang J, Wei X and Song B: Crosstalk between macrophages and astrocytes affects proliferation, reactive phenotype and inflammatory response, suggesting a role during reactive gliosis following spinal cord injury. J Neuroinflammation 12 : $109,2015$.

32. Impellizzeri D, Ahmad A, Di Paola R, Campolo M, Navarra M, Esposito E and Cuzzocrea S: Role of Toll like receptor 4 signaling pathway in the secondary damage induced by experimental spinal cord injury. Immunobiology 220: 1039-1049, 2015.

33. Ishii T Ueyama T, Shigyo M, Kohta M, Kondoh T, Kuboyama T, Uebi T, Hamada T, Gutmann DH, Aiba A, et al: A novel rac1-gspt1 signaling pathway controls astrogliosis following central nervous system injury. J Biol Chem 292: 1240-1250, 2017.

34. Ruzicka J, Machova-Urdzikova L, Gillick J, Amemori T, Romanyuk N, Karova K, Zaviskova K, Dubisova J, Kubinova S, Murali R, et al: A comparative study of three different types of stem cells for treatment of rat spinal cord injury. Cell Transplant 26: 585-603, 2017

35. Tang L, Lu X, Zhu R, Qian T, Tao Y, Li K, Zheng J, Zhao P, Li S, Wang X and Li L: Adipose-derived stem cells expressing the neurogenin-2 promote functional recovery after spinal cord injury in rat. Cell Mol Neurobiol 36: 657-667, 2016.

36. Liu XL, Zhang W and Tang SJ: Intracranial transplantation of human adipose-derived stem cells promotes the expression of neurotrophic factors and nerve repair in rats of cerebral ischemia-reperfusion injury. Int J Clin Exp Pathol 7: 174-183, 2014.

37. Menezes K, Nascimento MA, Goncalves JP, Cruz AS, Lopes DV, Curzio B, Bonamino M, de Menezes JR, Borojevic R, Rossi MI and Coelho-Sampaio T: Human mesenchymal cells from adipose tissue deposit laminin and promote regeneration of injured spinal cord in rats. PloS one 9: e96020, 2014.

38. Yeh DC, Chan TM, Harn HJ, Chiou TW, Chen HS, Lin ZS and Lin SZ: Adipose tissue-derived stem cells in neural regenerative medicine. Cell Transplant 24: 487-492, 2015.

39. Yang YC, Liu BS, Shen CC, Lin CH, Chiao MT and Cheng HC: Transplantation of adipose tissue-derived stem cells for treatment of focal cerebral ischemia. Curr Neurovasc Res 8: 1-13, 2011.

This work is licensed under a Creative Commons Attribution-NonCommercial-NoDerivatives 4.0 International (CC BY-NC-ND 4.0) License. 